\title{
Development and evaluation of 4 loop-mediated isothermal amplification assays to detect mastitis-causing bacteria in bovine milk samples
}

\author{
Karien Griffioen, ${ }^{1 *}$ † Jan Cornelissen, ${ }^{2 *}$ Annet Heuvelink, ${ }^{3}$ Daniela Adusei, ${ }^{2}$ Dik Mevius, ${ }^{2,4}$ \\ Fimme Jan van der Wal, ${ }^{2}$ and 1 Health4Food-Dutch Mastitis Diagnostics Consortium $\ddagger$ \\ ${ }^{1}$ Faculty of Veterinary Medicine, Department of Farm Animal Health, Utrecht University, PO Box 80151, 3508 TD Utrecht, the Netherlands \\ ${ }^{2}$ Wageningen Bioveterinary Research, Department of Infection Biology, Wageningen UR, PO Box 65, 8200 AB Lelystad, the Netherlands \\ ${ }^{3}$ Royal GD, PO Box 9, 7400 AA Deventer, the Netherlands \\ ${ }^{4}$ Department of Infectious Diseases and Immunology, Faculty of Veterinary Medicine, Utrecht University, PO Box 80165, 3508 TD Utrecht, \\ the Netherlands
}

\section{ABSTRACT}

Farmers prefer fast, sensitive, and on-site tests for treatment decisions on mastitis. Due to the time to results of the currently available diagnostic tools, these are rarely used for that purpose. Genotypic tests that do not require a growth step may be suitable for onsite testing, for example loop-mediated isothermal amplification (LAMP), which has been described as a sensitive test that can be used on-site. Therefore, this study aimed to develop and evaluate LAMP assays for the detection of a subset of mastitis-causing pathogens, Escherichia coli, Klebsiella pneumoniae, Staphylococcus aureus, and Streptococcus spp., in milk from cows with clinical mastitis. Furthermore, a generic nucleic acid lateral flow immunoassay (NALFIA) was evaluated as a potential on-site readout of the LAMP assays. For each assay of LAMP and NALFIA, the limit of detection and analytical specificity were determined using isolates, and the diagnostic specificity was determined using selected samples with known etiology. In addition, the diagnostic specificity of LAMP was determined using field samples with unknown etiology at testing. Bacteriological culture with identification by mass spectrometry was used as a reference method. The 4 assays had a kappa $\geq 0.73$ with the reference method when testing the selected samples, but $\geq 0.47$ when testing field samples. After correcting for prevalence, kappa was $\geq 0.80$ for the E. coli, K. pneumoniae, and Staph. aureus assays. The Streptococcus spp. assay had a kappa of 0.47 (0.48 after correction) with the ref-

Received December 10, 2019.

Accepted April 6, 2020.

*These authors contributed equally to this work.

†Corresponding author: k.griffioen@gddiergezondheid.nl

$\ddagger$ This consortium also includes R. P. Achterberg (Wageningen Bioveterinary Research, Lelystad, the Netherlands) and C. G. Scherpenzeel, M. M. C. Holstege, T. J. G. M. Lam, A. G. J. Velthuis, and R. Dijkman (GD Animal Health, Deventer, the Netherlands). erence method, probably caused by the assay broadly targeting a genus instead of a particular species. The NALFIA readout was found to have kappa $\geq 0.81$ for the E. coli, Staph. aureus, and Streptococcus spp. assays at a generic runtime, but for the K. pneumoniae assay a shorter runtime could be used. In conclusion, LAMP is a promising method for fast on-site tests for mastitiscausing pathogens if the current elaborate method for sample preparation is replaced by a simplified protocol. The NALFIA is an easy and reliable readout for on-site use, with the observation that for the current assay designs a generic runtime is not yet possible for the chosen set of pathogens. If associated with a simple and fast sample preparation protocol, the combination of LAMP and NALFIA has the potential to enable fast and reliable on-site testing of clinical mastitis milk samples.

Key words: dairy cattle, loop-mediated isothermal amplification, on-site mastitis test, nucleic acid lateral flow assay

\section{INTRODUCTION}

Mastitis is a common disease on dairies and has the highest contribution to the usage of antimicrobials in dairy cows (Pol and Ruegg, 2007; Kuipers et al., 2016). Currently, the decision to treat clinical mastitis with antimicrobials is mostly based on clinical signs (Sears and McCarthy, 2003; Griffioen et al., 2016), regardless of the actual cause. To enable targeted treatments, rapid and specific diagnostic tests to identify the cause of mastitis would be of added value.

Two types of diagnostic tests are frequently used for pathogen identification in mastitis: phenotypic and genotypic (Duarte et al., 2015). The advantage of genotypic tests over phenotypic tests is that the first type has a short time to result, provided that the test can be applied directly on the sample and no growth step is necessary. Furthermore, genotypic tests are more sensi- 
tive than (growth-dependent) phenotypic tests, as they have the ability to detect DNA of dead, or less viable, pathogens (Lam et al., 2009; Duarte et al., 2015). A disadvantage of genotypic tests is that if detected DNA originates from dead pathogens, a positive test result does not necessarily indicate an active IMI. Genotypic tests such as real-time PCR are used in diagnostic laboratories to detect various mastitis pathogens (Koskinen et al., 2009, 2010; Shome et al., 2011; Mahmmod et al., 2013), but are not frequently used in practice (Griffioen et al., 2016). Dutch farmers indicated that they are willing to use diagnostic tests more often if a simple test would be accessible close by, rather than at a distant laboratory (Griffioen et al., 2016). As expensive equipment is required to perform real-time PCR, these tests are performed in specialized diagnostic laboratories and therefore require time for transport of samples. Thus, even though available real-time PCR tests are quick, the time to result is too long to comply with the farmers' demands for treatment decisions. Therefore, less cumbersome options than real-time PCR, which can be performed at a veterinary clinic or on-farm, may fulfill the need of dairy farmers and may support incorporating diagnostic information into the decisionmaking process of mastitis treatments.

A technique that is advocated for on-site DNA amplification to detect pathogens, is loop-mediated isothermal amplification (LAMP; Mori and Notomi, 2009; Ashraf and Imran, 2018). This technique has the advantage over (real-time) PCR in that expensive equipment for temperature cycling and detection of results is not required, and that the conditions for the extraction and purification of DNA are less demanding (Kaneko et al., 2007; Sowmya et al., 2012). These advantages have resulted in LAMP tests for on-site use to detect Staphylococcus aureus in food samples (Tian et al., 2018), and Bergomoviruses under field conditions, using a portable device (Tian et al., 2018; Wilisiani et al., 2019).

Several studies describe the ability of LAMP to detect pathogens in milk samples (Song et al., 2012; Yang et al., 2014; Sathish et al., 2016; Appelt et al., 2019; Sange et al., 2019). Numerous LAMP assays, for example those developed to detect Staph. aureus and Streptococcus uberis, have a time to result of only 1 to 2 $\mathrm{h}$ and a high diagnostic accuracy (Tie et al., 2012; Cornelissen et al., 2016; Sheet et al., 2016). Thus, LAMP seems to be a promising method for on-site detection of mastitis-causing pathogens, and has the potential to fulfill the need of dairy farmers to obtain results within a short time. Therefore, this study aimed to develop and evaluate LAMP assays to detect a subset of mastitis-causing pathogens (Escherichia coli, Klebsiella pneumoniae, Staph. aureus, and Streptococcus spp.) in milk from cows with clinical mastitis, and to evaluate a generic nucleic acid lateral flow immunoassay (NALFIA) as a potential on-site readout of LAMP.

\section{MATERIALS AND METHODS}

\section{LAMP Assay Design}

Four new LAMP assays were designed targeting genes described in literature as valid targets for detection of E. coli: PhoA gene (Shome et al., 2011), K. pneumoniae: UreD gene (Zamani et al., 2013), Staph. aureus: nuc gene (Tie et al., 2012), and Streptococcus spp.: $16 S$ rRNA gene (Wang and Liu, 2015). The gene sequences were retrieved from the National Center for Biotechnology Information (NCBI) database (http:/ /www.ncbi.nlm.nih.gov; accession numbers FJ546461, L07039, EF529606, and AP011114.1, respectively). Conserved regions were identified by sequence alignments using NBCI Blast search (http://blast.ncbi.nlm .nih.gov), and were used for primer design with LAMP Designer 1.14 (Premier Biosoft, Palo Alto, CA). All LAMP assays were designed with 6 primers: 2 outer primers, 2 inner primers, and 2 loop primers (Table 1 ).

\section{Evaluation of LAMP Assays}

The evaluation process of the 4 LAMP assays consisted of 4 steps. First, the limit of detection was determined using dilution series of 1 isolate of the target bacterium. Second, the analytical specificity (Sp) was determined using a fixed set of isolates of different bacterial species. Thereafter, the diagnostic Sp was determined using 4 sets of selected milk samples, each compiled to allow evaluation of a specific LAMP assay. The last step was to determine the diagnostic performance by testing 163 milk samples with unknown etiology at the time of testing. These samples originated from cows with clinical mastitis and were collected in a field study for this purpose.

Bacterial Isolates and Milk Samples. To determine the limit of detection for each LAMP assay, 4 positive controls were selected and prepared as described (Cornelissen et al., 2016). The positive controls were $E$. coli strain 13-L24, K. pneumoniae strain 2.35, Staph. aureus strain 2.24, and S. uberis strain 2.28. Cultures of these isolates were serial diluted 5-fold in milk, starting with a 1:5 dilution. The E. coli suspension started with a counting of $8.0 \times 10^{7}\left(\mathrm{SD} 2.8 \times 10^{7}\right) \mathrm{cfu} / \mathrm{mL}$, K. pneumoniae with a counting of $5.0 \times 10^{8}(\mathrm{SD} 8.5 \times$ $\left.10^{7}\right) \mathrm{cfu} / \mathrm{mL}$, Staph. aureus with a counting of $1.1 \times 10^{8}$ (SD $1.4 \times 10^{7}$ ) cfu $/ \mathrm{mL}$, and S. uberis with a counting of $3.9 \times 10^{8}\left(\mathrm{SD} 1.4 \times 10^{7}\right) \mathrm{cfu} / \mathrm{mL}$. The number of $\mathrm{cfu}$ per $\mathrm{mL}$ was determined by serial dilution and plating 
in duplicate on sheep blood agar heart infusion plates (Wageningen Bioveterinary Research, Lelystad, the Netherlands). The milk originated from healthy cows and was checked to be culture negative for the 4 target pathogens.

Second, isolates from different bacterial species (Table 2), all verified using MALDI-TOF MS (see below), were used to determine the analytical Sp of the LAMP assays. The isolates are from an in-house collection (Cornelissen et al., 2016); a large subset originates from cows with mastitis, a smaller subset from other relevant species. The isolates were cultured and harvested as described (Cornelissen et al., 2016).

The first set of milk samples, used to determine the diagnostic Sp, was a subset of the milk samples used in the study described by Griffioen et al. (2018) and will be referred to as selected milk samples. In that study, 866 milk samples were analyzed at the bacteriological laboratory of Royal GD (Deventer, the Netherlands) by the reference method [i.e., bacteriological culture with identification using MS (see below)] between April and July 2016. Subsets were compiled with culture-positive and culture-negative samples for each target of the 4 LAMP assays (Table 2).
The second set of milk samples was tested without knowing the etiology at moment of testing in all 4 LAMP assays to determine the diagnostic performance. The set consisted of 163 milk samples from cows with mild and moderate clinical mastitis collected in a field study. These field samples were collected on 15 dairy farms in the Netherlands between May 2017 and July 2018 (unpublished data). The field samples were also analyzed at the bacteriological laboratory of GD Animal Health (Deventer, the Netherlands) by the reference method (Table 2; see below).

Reference Method. Bacteriological culture with identification using MS, as performed at the routine bacteriology laboratory of Royal GD, was used as reference method (Griffioen et al., 2018). In short, $10 \mu \mathrm{L}$ of milk was inoculated onto $6 \%$ sheep blood agar plates to determine the bacteriological status. Plates were incubated aerobically for $48 \mathrm{~h}$ at $37^{\circ} \mathrm{C}$. Growth of presumptive mastitis-causing bacteria was examined after 24 and $48 \mathrm{~h}$, according to the guidelines of the National Mastitis Council (NMC, 1999). Presumptive mastitiscausing bacteria were identified using MALDI-TOF MS (MALDI Biotyper 3.1, Bruker Daltonics GmbH, Bremen, Germany; Barreiro et al., 2010).

Table 1. Sequences of 4 designed loop-mediated isothermal amplification (LAMP) primers ${ }^{1}$

\begin{tabular}{|c|c|c|c|}
\hline LAMP assay & Gene & Primer & Sequence $\left(5^{\prime}-3^{\prime}\right)$ \\
\hline \multirow{4}{*}{ Escherichia coli } & \multirow{4}{*}{$P h o A^{2}$} & Ecoli 4 B3 & CTTTGCTGAAACGGCAAC \\
\hline & & Ecoli $4 \mathrm{FIP}^{3}$ & CTGACGGCAATATGCCAGTGATCGATATTGCCATGGTACG \\
\hline & & Ecoli 4 Floop & GCTGGCTAGGACCGAAAG \\
\hline & & Ecoli 4 Bloop & TTCAGTGAGGCAGCATCG \\
\hline \multirow[t]{3}{*}{ Klebsiella pneumoniae } & \multirow[t]{3}{*}{$U r e D^{5}$} & Kleb 3 F3 & GATCTCCGCTTTCAGCAG \\
\hline & & Kleb 3 Floop $^{4}$ & GAAGCAGATAGAGGTGACAGG \\
\hline & & Kleb 3 Bloop $^{3}$ & CTGCCATACGCTGATAACCA \\
\hline \multirow[t]{4}{*}{ Staphylococcus aureus } & \multirow[t]{4}{*}{$n u c^{6}$} & SA NUC F3-2 & AACAGTATATAGTGCAACTTCAA \\
\hline & & SA NUC B3-2 & CTTTGTCAAACTCGACTTCAA \\
\hline & & SA NUC FIP- $2^{3}$ & TGTCATTGGTTGACCTTTGTACATTAAAATTACATAAAGAACCTGCGA \\
\hline & & SA NUC BIP-2 $2^{4}$ & GTTGATACACCTGAAACAAAGCATCATTTTTTTCGTAAATGCACTTGC \\
\hline \multirow{3}{*}{ Streptococcus spp. } & \multirow{3}{*}{$16 S r R N A^{7}$} & Strep $4 \mathrm{BIP}^{4}$ & TGGTTGTTACAACGAGTCGCAATCCGAACTGAGATTGTC \\
\hline & & Strep 3 Floop & TTATTACCGGCAGTCTCGC \\
\hline & & Strep 3 Bloop & TGACGGCAAGCTAATCTCTTAA \\
\hline
\end{tabular}

${ }^{1}$ Ecoli $=$ E. coli Kleb $=$ K. pneumoniae $; \mathrm{SA}=$ Staph. aureus $;$ Strep $=$ Streptococcus $\mathrm{spp} . ; \mathrm{FIP}=$ forward inner primer; $\mathrm{BIP}=$ backward inner primer; Floop $=$ forward loop primer; Bloop $=$ backward loop primer; NUC $=n u c$.

${ }^{2}$ Assay designed using target gene as described by Shome et al. (2011).

${ }^{3}$ Primer labeled with FAM when used in nucleic acid lateral flow immunoassay.

${ }^{4}$ Primer labeled with biotin when used in nucleic acid lateral flow immunoassay.

${ }^{5}$ Assay designed using target gene as described by Zamani et al. (2013).

${ }^{6}$ Assay designed using target gene as described by Tie et al. (2012).

${ }^{7}$ Assay designed using target gene as described by Wang and Li (2015). 
Griffioen et al.: DETECTION OF MASTITIS-CAUSING BACTERIA

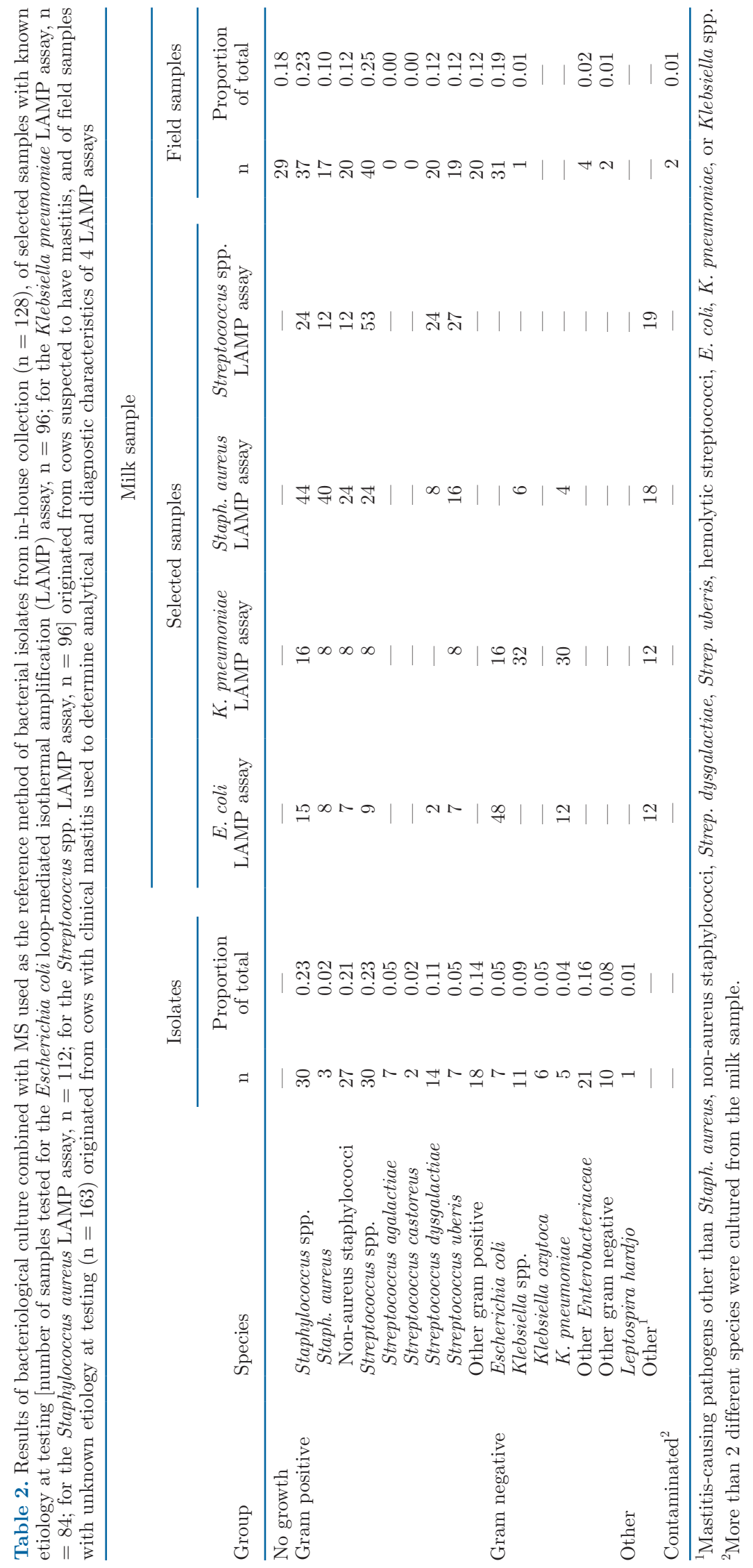


Isolation of DNA. Aliquots of $250 \mu \mathrm{L}$ of suspended bacteria from the isolates, or $500 \mu \mathrm{L}$ of milk samples, were used to isolate template DNA, essentially as described (Cornelissen et al., 2016). The bacterial suspensions and milk samples were incubated for $1 \mathrm{~h}$ at $37^{\circ} \mathrm{C}$ with $80 \mu \mathrm{L}$ of Tris-EDTA buffer $[20 \mathrm{mmol} / \mathrm{L}$ Tris- $\mathrm{HCl}$ ( $\mathrm{pH} 8.0$ ), and $2 \mathrm{mmol} / \mathrm{L}$ EDTA], containing achromopeptidase $(1,000 \mathrm{U} / \mathrm{mL})$, lysostaphin $(20 \mu \mathrm{L} /$ $\mathrm{mL})$, lysozyme $1(\mathrm{mg} / \mathrm{mL})$, and mutanolysin $100(\mathrm{U} /$ $\mathrm{mL}$; Sigma-Aldrich Chemie, Zwijndrecht, the Netherlands). Thereafter, $40 \mu \mathrm{L}$ of proteinase, $360 \mu \mathrm{L}$ of ATL buffer from the DNeasy Blood and Tissue Kit (Qiagen, Venlo, the Netherlands), and $400 \mu \mathrm{L}$ of AL buffer from the same kit was added, and the mix was incubated for $1 \mathrm{~h}$ at $56^{\circ} \mathrm{C}$. Of a mixture of $400 \mu \mathrm{L}$ of ethanol and $3 \mu \mathrm{L}$ of $\mathrm{HCl}(25 \%), 400 \mu \mathrm{L}$ was added to the mix. Membranes were washed according to the guidelines of the manufacturer and the isolated DNA was eluted with $50 \mu \mathrm{L}$ of water.

LAMP Reactions. For each LAMP assay, positive controls were used at a concentration of $1 \mathrm{ng} / \mu \mathrm{L}$. For the analytical performance, $2 \mu \mathrm{L}$ of the positive controls was used, and for the diagnostic performance, 8 $\mu \mathrm{L}$ was used.

Loop-mediated isothermal amplification reactions were in essence performed as described (Cornelissen et al., 2016), using a commercially available mix with a polymerase with strand displacement activity and EvaGreen (Isothermal Mastermix, OptiGene, Horsham, UK). Standard desalted oligonucleotides were used, synthesized on a $40 \mathrm{nmol}$ scale (Biolegio, Nijmegen, the Netherlands). For E. coli, K. pneumoniae, and Staph. aureus, the LAMP reactions were performed at a final volume of $23.0 \mu \mathrm{L}$, containing the Isothermal Mastermix, $2.0 \mu \mathrm{mol} / \mathrm{L}$ each of the forward inner primer and the backward inner primer, $0.2 \mu \mathrm{mol} / \mathrm{L}$ each of the outer primers F3 and B3, $1.0 \mu \mathrm{mol} / \mathrm{L}$ each of the backward loop and forward loop primer, template DNA $(2 \mu \mathrm{L}$ if isolates were used or $8 \mu \mathrm{L}$ of eluate if milk samples were used), and $6 \mu \mathrm{L}$ of ROX Passive reference (Eurogentec, Liege, Belgium). For Streptococcus spp., the LAMP reactions were performed at a final volume of $18.5 \mu \mathrm{L}$, containing the Isothermal Mastermix, $0.8 \mu \mathrm{mol} / \mathrm{L}$ each of the forward inner primer and the backward inner primer, $0.2 \mu \mathrm{mol} / \mathrm{L}$ each of $\mathrm{F} 3$ and B3 primers, $0.4 \mu \mathrm{mol} / \mathrm{L}$ each of the backward loop and forward loop primer, template DNA $(2 \mu \mathrm{L}$ if isolates were used or $8 \mu \mathrm{L}$ of template DNA if milk samples were used), and $6 \mu \mathrm{L}$ of ROX Passive reference. The LAMP assays were performed in 96-well plates and always included negative controls (water) and positive controls. Reactions were incubated at $62^{\circ} \mathrm{C}$ in an $\mathrm{ABI}$ 7500 Real-Time PCR system (Applied Biosystems,
Foster City, CA) for 90 cycles of $45 \mathrm{~s}$, and thus in total for $67.5 \mathrm{~min}$. The LAMP products were detected by screening for fluorescence by EvaGreen, which is referred to as the real-time readout. The threshold was manually set at $50 \%$ in the linear phase of the amplification plot of the positive control, similar to the selection of a threshold for real-time PCR (Caraguel et al., 2011). The time point at which fluorescent signals passed this threshold is referred to as time-to-positivity (Tp) and is expressed in minutes. To stop the reaction, the temperature was increased to $95^{\circ} \mathrm{C}$ to inactivate the polymerase. To confirm the identity of amplification products, melting curve analyses were performed after amplification by decreasing the temperature from $95^{\circ} \mathrm{C}$ to $60^{\circ} \mathrm{C}$ during which fluorescence was measured (see below for details on the analysis). Finally, to establish if actual amplification had taken place, for each sample the presence of a sigmoidal curve was checked.

LAMP Using NALFIA. To determine whether LAMP has potential to be used on-site, a lateral-flowbased readout that does not require equipment (i.e., NALFIA) was evaluated. To enable readout by NALFIA, FAM- and biotin-labeled amplicons were generated in the LAMP reactions. For that, in each primer set, 2 unlabeled primers were replaced with a FAM- and biotin-labeled version, respectively (Table 2). From a practical point of view, a generic assay time was chosen for all LAMP assays to stop the reactions in the realtime PCR machine by increasing the temperature to $95^{\circ} \mathrm{C}$, which was $40 \mathrm{~min}$, based on the Tp with the highest total area under the receiver operator (ROC) curve (AUC) for all assays. The reaction mixtures of the experiment were taken up in reaction tubes that were inserted in contamination-free cassettes (U-Star Disposable Nucleic Acid Lateral Flow Detection Units, TwistDX Limited, Maidenhead, UK). These cassettes enable analysis of labeled amplification products by NALFIA. The cassettes contain a needle to perforate a reaction tube upon insertion, and a reservoir in which amplification products mix with streptavidinconjugated colloidal gold. This results in labeling of biotinylated amplicons from the LAMP reaction with red gold particles. The resulting mixture flows into the connected nitrocellulose strip along immobilized anti-FAM antibodies, where, upon interaction with red gold-amplicon-FAM complexes, amplicons are visualized without the need for special equipment.

\section{Analytical and Diagnostic Performance of LAMP Assays}

Limit of Detection. To determine the limit of detection of the LAMP assays, each spiked milk sample 
from the 5 -fold dilution series was tested in 3 independent experiments. The limit of detection was calculated as the lowest mean concentration of bacteria where all 3 experiments gave a signal within the length of the experiment plus 3 times standard deviation.

Analytical Specificity. To determine the analytical $\mathrm{Sp}$, the cut-off for the Tp was defined as the average time it took for signals to appear when testing nontarget isolates, minus 3 times standard deviation, using only data on signals that appeared within the length of the experiment. Isolates with a Tp lower than the calculated Tp cut-off were considered positive.

Diagnostic Performance of LAMP Using Selected Milk Samples. To determine the diagnostic performance of LAMP in the selected milk samples, Tp cut-offs for each LAMP assay were determined for each minute during the running time of the experiment using ROC analyses in Stata Statistical Software (StataCorp, 2017, Release 15, StataCorp LLC, College Station, TX). The AUC was established using binary results of all samples (i.e., positive or negative at the tested time points), based on the presence or absence of a valid signal. Accompanying $95 \%$ confidence intervals were determined around the AUC. A valid signal was defined as a putative positive sample (i.e., a sample with a signal) with the melting temperature (Tm) of the product within the Tm range, calculated as the mean Tm of the positive controls plus or minus a margin of $2^{\circ} \mathrm{C}$, and with a sigmoidal amplification curve. Samples with aberrant Tm or amplification curves were deemed negative and ignored for Tp cut-off calculations, but the results were used for calculating assay sensitivity (Se) and Sp. The Tp resulting in the highest AUC was selected as Tp cut-off. Further, to calculate the test Se and Sp, samples were designated as positive or negative according to the determined Tp cut-off, and samples with an aberrant Tm or sigmoidal curve were included as negative results.

Diagnostic Performance of LAMP Using Clinical Mastitis Milk Field Samples. The diagnostic performance of the LAMP assays using field samples was determined using the 163 clinical mastitis samples. Each result was judged positive or negative based on the 3 criteria, using the Tp cut-off as determined with ROC analysis over the selected samples, the Tm, and whether a sigmoidal curve was observed.

\section{Statistical Analysis}

Results of each LAMP assay and both types of readout were compared with results of the reference method to calculate the diagnostic test characteristics Se, defined as the proportion of culture-positive samples with a positive LAMP result, and Sp, defined as the proportion of culture-negative samples with a negative LAMP result (i.e., no signal below the Tp threshold, an aberrant Tm, or an aberrant amplification curve), with the accompanying binomial exact $95 \%$ CI at all steps of the evaluation (Dohoo et al., 2009). Furthermore, the proportion of agreement between the reference method and LAMP assays corrected for chance was calculated, known as Cohen's kappa ( $\kappa)$. In addition to $\kappa$, also the prevalence- and bias-adjusted kappa (PABAK) was calculated (Byrt et al., 1993) for the field samples. The guideline of Landis and Koch (1977) was used to determine the magnitude of agreement. A value of $>0.80$ was considered to be almost perfect, $>0.60-0.80$ substantial, $>0.40-0.60$ moderate, $>0.20-0.40$ fair, $>0.00-0.20$ slight, and $\leq 0.00$ poor agreement.

\section{RESULTS}

\section{Limit of Detection}

The limit of detection for both readouts were equal for all LAMP assays, except for the E. coli LAMP assay where the limit of detection of the real-time readout was lower (Table 3). The Streptococcus spp. LAMP assay was the most sensitive assay with $1.1 \times 10^{3} \mathrm{cfu} / \mathrm{mL}$ as detection limit for both readouts.

\section{Analytical Specificity Using Isolates}

The analytical specificity determination showed that all LAMP assays had high Se and Sp $(\geq 0.96)$. Also, kappa was substantial to almost perfect for the 4 assays to detect their target bacteria (Table 4). At this step of the evaluation, the calculated Tp cut-off for the $E$. coli LAMP assay was $38 \mathrm{~min}$. The observed Tp values for E. coli isolates ranged from 14 to 24 min (mean 17 min). The isolate for which a false-positive result was found was an Enterococcus durans with a Tp of $36 \mathrm{~min}$. For the K. pneumoniae assay, the calculated Tp cut-off was $25 \mathrm{~min}$. The observed $\mathrm{Tp}$ values for $K$. pneumoniae isolates ranged from 7 to $9 \mathrm{~min}$ (mean $8 \mathrm{~min}$ ). The isolates for which false-positive results were found were 1 E. durans and $4 \mathrm{~K}$. oxytoca isolates, which had a Tp between 17 and $24 \mathrm{~min}$. For the Staph. aureus assay, the calculated Tp cut-off was $18 \mathrm{~min}$. The observed Tp for Staph. aureus isolates ranged from 6 to $7 \mathrm{~min}$. For the Streptococcus spp. assay, the calculated Tp cut-off was 15 min. The observed Tp for Streptococcus spp. isolates ranged from 9 to 11 min (mean $10 \mathrm{~min}$ ). The latter 2 assays had only true-positive and true-negative results. 


\section{Diagnostic Performance of LAMP Using Selected Milk Samples}

The Tp cut-offs for each LAMP assay as obtained with the ROC analysis are shown in Table 5. If multiple time points were found to have the highest AUC, the point with the lowest Tp value was chosen. Applying the optimal Tp cut-off to the whole set of selected samples resulted in Se ranging from 0.73 to 0.98 , and Sp ranging from 0.84 to 0.96 for all LAMP assays (Table 6).

At the optimal Tp cut-off of $31 \mathrm{~min}$ for the E. coli assay, Se was 0.83 (Table 6 ). The Tp of true-positive samples ranged from 15 to 31 min (mean $22 \mathrm{~min}$ ). The average $\mathrm{Tm}$ of the positive controls was $89.0^{\circ} \mathrm{C}$. Of the samples with false-negative results, 6 samples did not give a signal within the length of the experiment or above the optimal Tp cut-off, and 2 had a Tm below the Tm range (average $\mathrm{Tm} 86.5^{\circ} \mathrm{C}$ ). The 4 samples with false-positive results were K. pneumoniae, Staph. aureus, S. uberis, and an unspecified bacterium [growth, but no Staph. aureus, K. pneumoniae, E. coli, non-aureus staphylococci (NAS), or Streptococcus spp.] according to the reference method.

At the optimal Tp cut-off of $13 \mathrm{~min}$ for the K. pneumoniae assay, Se was 0.73 (Table 6). The Tp of the samples with true-positive results ranged from 7 to 12 min (mean $8 \mathrm{~min}$ ). The average Tm was $90.9^{\circ} \mathrm{C}$. Seven of the samples that were determined to have falsenegative results had no signal within the length of the experiment or the Tp was too high, and 1 had Tm of $88.8^{\circ} \mathrm{C}$. The 2 samples with false-positive results were E. coli, and NAS according to the reference method.

At the optimal Tp cut-off of $26 \mathrm{~min}$ for the Staph. aureus LAMP assay, Se was 0.83 (Table 6). The Tp of the samples with positive results ranged from 6 to 25 min (mean $11 \mathrm{~min}$ ). The average $\mathrm{Tm}$ of the positive controls was $81.1^{\circ} \mathrm{C}$. The samples with false-negative results were scored negative based on the Tp being too high $(\mathrm{n}=6)$, and 1 had a $\mathrm{Tm}$ of $79.0^{\circ} \mathrm{C}$. The 4 samples with false-positive results were $S$. dysgalactiae, NAS (n $=2$ ), and an unspecified bacterium.
At the optimal Tp cut-off of 48 min for the Streptococcus spp. LAMP assay, Se was 0.98 (Table 6). The Tp ranged from 7 to $48 \mathrm{~min}$ (mean $29 \mathrm{~min}$ ). The average $\mathrm{Tm}$ of the true positives was $87.6^{\circ} \mathrm{C}$. The sample with the false-negative result scored negative because no signal was detected within the length of the experiment. Of the samples with false-positive results, 1 was positive for NAS, and 6 contained unspecified bacteria according to the reference method.

\section{Diagnostic Performance of LAMP Using Clinical Mastitis Milk Samples}

The diagnostic performance of the 4 LAMP assays using 163 field samples is shown in Table 4. The Se for all assays ranged from 0.55 to 1.00 , and Sp from 0.68 to 1.00 . Kappa ranged from 0.47 to 0.81 , but was often affected by prevalence and bias as shown by PABAK.

For the E. coli assay the observed Tp of the samples with true-positive results ranged from 17 to $30 \mathrm{~min}$ (mean $23 \mathrm{~min}$ ). The samples deemed false positive by LAMP were $S$. uberis according to the reference method. Of the positive samples according to the reference method, 14 were deemed negative by LAMP based on the Tp being too high or not detected $(\mathrm{n}=13)$, or because the Tm was outside of the Tm range of 86.3 to $90.3^{\circ} \mathrm{C}\left(\mathrm{n}=1 ; 75.3^{\circ} \mathrm{C}\right)$.

Only 1 field sample was positive for $K$. pneumoniae according to the reference method, for which the $K$. pneumoniae LAMP assay was also positive. Two samples were deemed false positive by LAMP, one from which Bacillus pumilus was recovered according to the reference method, and for the other no growth was reported.

For the Staph. aureus assay the observed Tp of the samples with true-positive results ranged from 7 to 15 min (mean $11 \mathrm{~min}$ ). None of the samples was deemed as false positive by LAMP. Of the positive samples according to the reference method, 5 were deemed negative by LAMP because no signal was found.

Table 3. Lowest concentration detected, SD, and limit of detection (LOD; lowest concentration $+3 \times \mathrm{SD})$ in cfu/mL of 4 designed loopmediated isothermal amplification (LAMP) assays using 5-fold dilutions of target bacteria spiked in milk in 2 types of readouts: real-time readout of fluorescence in a PCR machine tested in 3 independent experiments and nucleic acid lateral flow immunoassays (NALFIA) tested in 1 experiment

\begin{tabular}{|c|c|c|c|c|c|c|c|}
\hline \multirow[b]{2}{*}{ Bacteria } & \multirow[b]{2}{*}{ Gene } & \multicolumn{3}{|c|}{ Real-time readout } & \multicolumn{3}{|c|}{ NALFIA readout } \\
\hline & & $\begin{array}{c}\text { Lowest } \\
\text { concentration }\end{array}$ & SD & LOD & $\begin{array}{c}\text { Lowest } \\
\text { concentration }\end{array}$ & SD & LOD \\
\hline Klebsiella pneumoniae & UreD & $1.3 \times 10^{3}$ & $2.2 \times 10^{2}$ & $1.9 \times 10^{3}$ & $1.3 \times 10^{3}$ & $2.2 \times 10^{2}$ & $1.9 \times 10^{3}$ \\
\hline Staphylococcus aureus & $n u c$ & $1.4 \times 10^{3}$ & $1.8 \times 10^{2}$ & $2.0 \times 10^{3}$ & $1.4 \times 10^{3}$ & $1.8 \times 10^{2}$ & $2.0 \times 10^{3}$ \\
\hline Streptococcus spp. & $16 S r R N A$ & $1.0 \times 10^{31}$ & $3.6 \times 10^{1}$ & $1.1 \times 10^{3}$ & $1.0 \times 10^{3}$ & $3.6 \times 10^{1}$ & $1.1 \times 10^{3}$ \\
\hline
\end{tabular}

${ }^{1}$ Tested in 2 independent experiments. 
Griffioen et al.: DETECTION OF MASTITIS-CAUSING BACTERIA

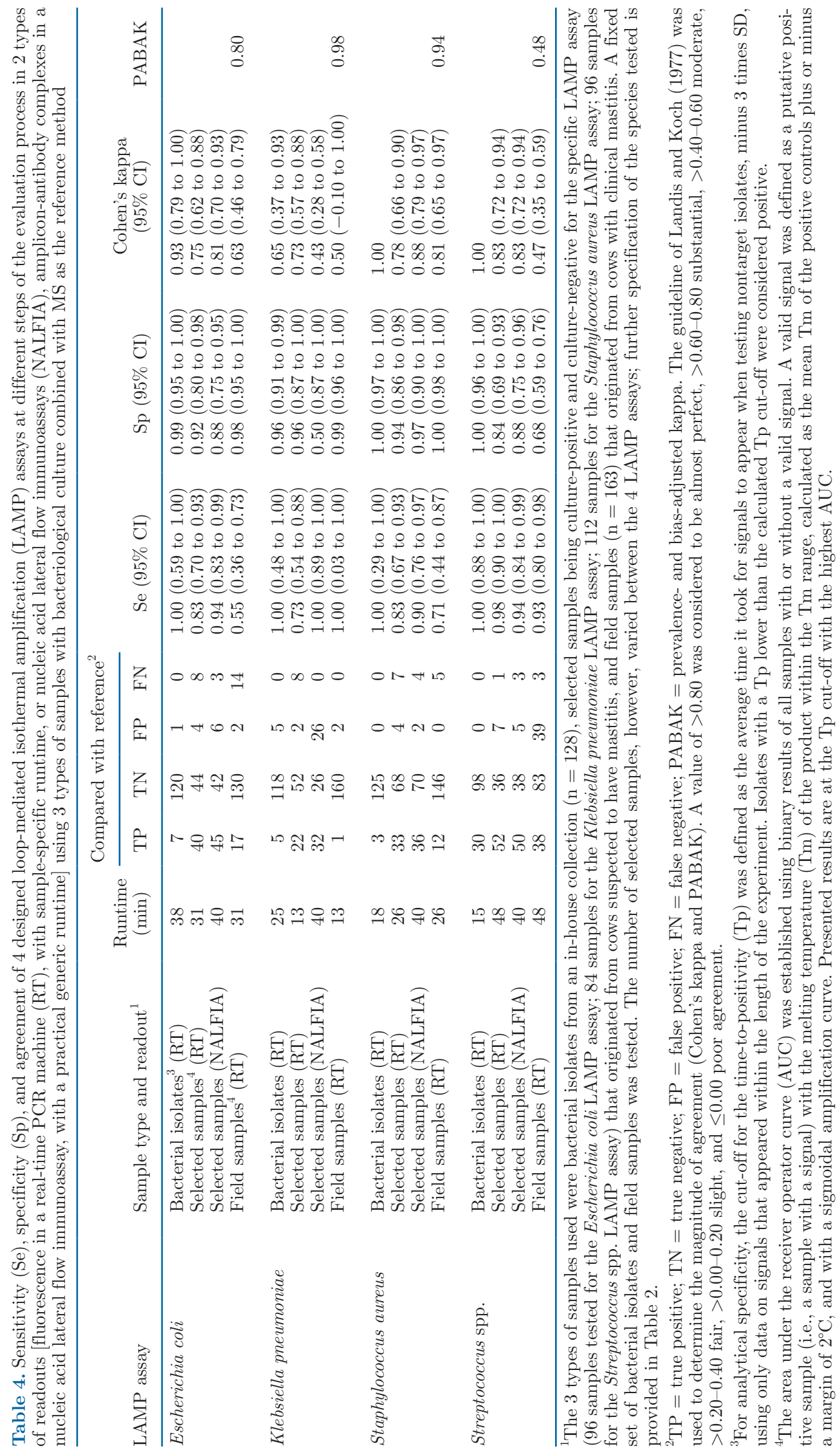


Table 5. Area under the receiver operator curve (AUC), with 95\% CI, of 4 loop-mediated isothermal amplification (LAMP) assays at different runtimes at which it was assessed whether a sample had a signal or not, to determine the optimal time-to-positivity cut-off (indicated with an asterisk $)^{1}$

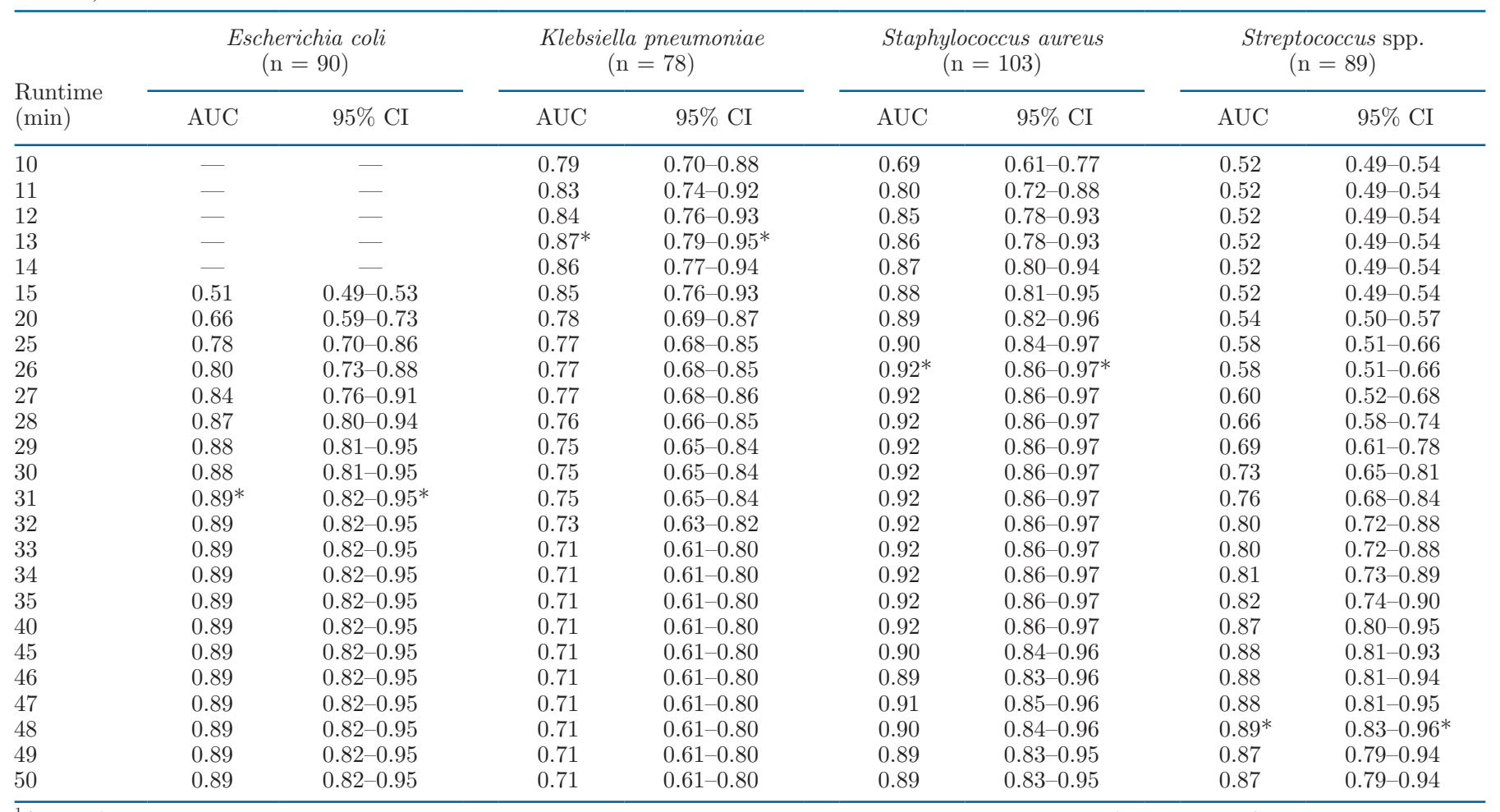

${ }^{1} \mathrm{~A}$ set of selected target positive and negative milk samples according to bacteriological culture with identification by MS were used that had a valid signal within $67.5 \mathrm{~min}$. Samples within the set that had an aberrant melting temperature based on the melting temperature of the positive controls or sigmoidal curve were excluded for this analysis. The sets contain samples with different bacterial species (tested species per assay are provided in Table 2); the number of samples tested is given for each set.

For the Streptococcus spp. assay, the observed Tp of the samples with true-positive results ranged from 6 to $47 \mathrm{~min}$ (mean $15 \mathrm{~min}$ ). The $\mathrm{Tp}$ of the samples with false-positive results ranged from 4 to 47 min (mean $23 \mathrm{~min}$ ). From the samples with false-positive results in the Streptococcus spp. assay, the reference method recovered no bacteria $(\mathrm{n}=9)$, NAS $(\mathrm{n}=9)$, E. coli $(\mathrm{n}$ $=5)$, Staph. aureus $(\mathrm{n}=4)$, Corynebacterium $\mathrm{spp} .(\mathrm{n}=$ 2 ), and the following pathogens were all detected once: Enterococcus saccharolyticus, K. pneumoniae, both NAS and Enterococcus faecalis, Staph. aureus and E. coli, E. coli and gram-positive cocci, Lactococcus garvieae, contamination, Serratia spp., Candida, or Enterococcus faecium. The Streptococcus spp. positive samples according to the reference method deemed negative by LAMP had a Tp above the cut-off $(\mathrm{n}=1)$, or no signal $(\mathrm{n}=2)$.

\section{NALFIA Readout}

Results of the NALFIA readout using the selected milk samples are shown in Table 4. A practical generic runtime of $40 \mathrm{~min}$ was selected based on the highest
AUC at that runtime over all assays using selected samples in the diagnostic performance analysis. The Se ranged from 0.90 to 1.00 over all LAMP assays, and the Sp ranged from 0.50 to 0.97 . For the samples with false-positive results in the $E$. coli assay, the reference method resulted in K. pneumoniae $(\mathrm{n}=5)$ and Staph. aureus $(\mathrm{n}=1)$. For the samples with false-positive results in the $K$. pneumoniae assay, the reference method resulted in E. coli $(\mathrm{n}=13)$, followed by Staph. aureus $(\mathrm{n}=5)$, NAS $(\mathrm{n}=4)$, S. uberis $(\mathrm{n}=1)$, and unspecified bacteria $(\mathrm{n}=3)$. For the samples with false-positive results in the Staph. aureus assay, the reference method resulted in $S$. dysgalactiae and an unspecified bacterium. For the samples with false-positive results in the Streptococcus spp. assay, the reference method resulted in NAS $(\mathrm{n}=2)$, Staph. aureus $(\mathrm{n}=1)$, and unspecified bacteria $(\mathrm{n}=2)$.

\section{DISCUSSION}

Fast, sensitive, and on-site tests are preferred by farmers to target their treatments in case of clinical mastitis (Griffioen et al., 2016). This study aimed to 
develop and to evaluate such assays. The 4 developed LAMP assays had high Se, Sp (both $\geq 0.73$ ), and kappa $(\geq 0.65)$ compared with the reference method to detect E. coli, K. pneumoniae, Staph. aureus, and Streptococcus spp., both for the isolates and for selected milk samples. For field samples, the Se was lower for 3 of 4 assays, but PABAK was $\geq 0.80$, except for the Streptococcus spp. assay. Considering the PABAK estimate for the E. coli, K. pneumoniae, and Staph. aureus LAMP assays, the agreement with the reference method was considered to be almost perfect. Most kappa estimates were influenced by the prevalence of the target bacteria in the field samples. In case of a low (or high) prevalence for the target of the assay, no high kappa values can be obtained as most of the results are deemed true positive or true negative (Byrt et al., 1993), and thus a skewed distribution of results would be obtained. Therefore, we also included PABAK in the results to correct for that effect, which is reflected most clearly for the K. pneumoniae assay used on the field samples, where the agreement changed from 0.50 (kappa) to 0.98
(PABAK) after correction for the low prevalence of $K$. pneumoniae.

The test performance of the LAMP assays using the isolates resembled the reference method, as shown by the high Se and Sp estimates. However, the assays were less sensitive (higher limit of detection) than the reference method, which has a limit of detection of approximately $100 \mathrm{cfu} / \mathrm{mL}$ (Ruegg, 2018). This probably caused the low Se found for the E. coli LAMP assay used on the field samples. Only one design was investigated; it is possible that in the chosen target region variation is larger than anticipated and that another design would result in a more sensitive assay. Most further evaluation steps for the evaluated assays showed high Se and Sp estimates compared with the reference method. However, 2 assays had low Sp, namely the $K$. pneumoniae assay using the NALFIA as readout on the selected milk samples, and the Streptococcus spp. assay using the fluorescence readout on the tested field samples. One explanation might be that there is a discrepancy between the library of the MALDI Biotyper

Table 6. Sensitivity (Se) and specificity (Sp) of 4 loop-mediated isothermal amplification (LAMP) assays at different runtimes ${ }^{1}$

\begin{tabular}{|c|c|c|c|c|c|c|c|c|}
\hline \multirow{2}{*}{$\begin{array}{l}\text { Runtime } \\
\text { (min) }\end{array}$} & \multicolumn{2}{|c|}{$\begin{array}{l}\text { Escherichia coli } \\
\qquad(\mathrm{n}=96)\end{array}$} & \multicolumn{2}{|c|}{$\begin{array}{l}\text { Klebsiella pneumoniae } \\
\qquad(\mathrm{n}=84)\end{array}$} & \multicolumn{2}{|c|}{$\begin{array}{c}\text { Staphylococcus aureus } \\
(\mathrm{n}=112)\end{array}$} & \multicolumn{2}{|c|}{$\begin{array}{l}\text { Streptococcus spp. } \\
(\mathrm{n}=96)\end{array}$} \\
\hline & $\mathrm{Se}$ & $\mathrm{Sp}$ & $\mathrm{Se}$ & $\mathrm{Sp}$ & $\mathrm{Se}$ & $\mathrm{Sp}$ & $\mathrm{Se}$ & $\mathrm{Sp}$ \\
\hline 10 & - & - & 0.57 & 1.00 & 0.35 & 1.00 & 0.04 & 1.00 \\
\hline 11 & - & - & 0.63 & 1.00 & 0.55 & 1.00 & 0.04 & 1.00 \\
\hline 12 & - & - & 0.67 & 1.00 & 0.65 & 1.00 & 0.04 & 1.00 \\
\hline 13 & - & - & $0.73^{*}$ & $0.98^{*}$ & 0.68 & 0.99 & 0.04 & 1.00 \\
\hline 14 & - & - & 0.77 & 0.93 & 0.70 & 0.99 & 0.04 & 1.00 \\
\hline 15 & 0.02 & 1.00 & 0.77 & 0.91 & 0.75 & 0.96 & 0.04 & 1.00 \\
\hline 20 & 0.31 & 1.00 & 0.80 & 0.76 & 0.78 & 0.94 & 0.08 & 1.00 \\
\hline 25 & 0.58 & 0.96 & 0.83 & 0.70 & 0.80 & 0.94 & 0.25 & 0.93 \\
\hline 26 & 0.63 & 0.96 & 0.83 & 0.70 & $0.83^{*}$ & $0.94^{*}$ & 0.25 & 0.93 \\
\hline 27 & 0.69 & 0.96 & 0.83 & 0.70 & 0.83 & 0.94 & 0.28 & 0.93 \\
\hline 28 & 0.75 & 0.96 & 0.83 & 0.69 & 0.83 & 0.94 & 0.40 & 0.93 \\
\hline 29 & 0.79 & 0.94 & 0.83 & 0.67 & 0.83 & 0.94 & 0.47 & 0.93 \\
\hline 30 & 0.79 & 0.94 & 0.83 & 0.67 & 0.83 & 0.94 & 0.55 & 0.93 \\
\hline 31 & $0.83^{*}$ & $0.92^{*}$ & 0.83 & 0.67 & 0.83 & 0.94 & 0.60 & 0.93 \\
\hline 32 & 0.83 & 0.92 & 0.83 & 0.63 & 0.83 & 0.94 & 0.68 & 0.93 \\
\hline 33 & 0.83 & 0.92 & 0.83 & 0.59 & 0.83 & 0.94 & 0.72 & 0.91 \\
\hline 34 & 0.85 & 0.90 & 0.83 & 0.59 & 0.83 & 0.94 & 0.75 & 0.88 \\
\hline 35 & 0.85 & 0.90 & 0.83 & 0.59 & 0.83 & 0.94 & 0.77 & 0.88 \\
\hline 40 & 0.85 & 0.90 & 0.83 & 0.59 & 0.83 & 0.94 & 0.94 & 0.84 \\
\hline 45 & 0.85 & 0.90 & 0.83 & 0.59 & 0.83 & 0.92 & 0.96 & 0.84 \\
\hline 46 & 0.85 & 0.90 & 0.83 & 0.59 & 0.83 & 0.90 & 0.96 & 0.84 \\
\hline 47 & 0.85 & 0.90 & 0.83 & 0.59 & 0.85 & 0.90 & 0.96 & 0.84 \\
\hline 48 & 0.85 & 0.90 & 0.83 & 0.59 & 0.85 & 0.89 & $0.98^{*}$ & $0.84^{*}$ \\
\hline 49 & 0.85 & 0.90 & 0.83 & 0.59 & 0.85 & 0.88 & 0.98 & 0.79 \\
\hline 50 & 0.85 & 0.90 & 0.83 & 0.59 & 0.85 & 0.88 & 0.98 & 0.79 \\
\hline 60 & 0.85 & 0.88 & 0.83 & 0.56 & 0.88 & 0.82 & 0.98 & 0.77 \\
\hline 70 & 0.85 & 0.83 & 0.87 & 0.56 & 0.88 & 0.79 & 0.98 & 0.74 \\
\hline
\end{tabular}

${ }^{1}$ To calculate Se and Sp for each possible runtime ( $\mathrm{min}$ ), the results of LAMP assays (absence or presence of a valid signal below the optimal time-to-positivity cut-off) for sets of selected milk samples were compared with results of the reference method (bacteriological culture with identification by MS). The sets contain samples with different bacterial species (tested species per assay are provided in Table 2); the number of samples tested is given for each set.

*The Se and Sp at the optimal time-to-positivity are indicated with asterisks. 
and the NCBI database; we found in the analytical specificity evaluation a $K$. pneumoniae positive result with the LAMP assay where this isolate was characterized as $K$. oxytoca. More likely is that the false-positive results for $K$. pneumoniae in the NALFIA readout were caused by the generic assay time we had chosen for all 4 assays, which was longer than the optimal Tp. In total, the AUC was highest for all 4 assays at $40 \mathrm{~min}$ and thus this Tp cut-off was selected and used as a practical generic runtime for the NALFIA readout. This runtime affected the results only slightly for the E. coli, Staph. aureus, and Streptococcus spp. assays. For K. pneumoniae, however, the runtime of $40 \mathrm{~min}$ increased Se, but lowered the Sp from 0.98 to 0.59 , and thus too many samples were deemed false positive, as compared with the reference method. Clearly, a generic runtime combined with NALFIA readout is only possible when the LAMP assays have a comparable Tp cut-off. For the present assays, that would require a (new) compatible LAMP assay for $K$. pneumoniae with a Tp cutoff around $40 \mathrm{~min}$. For the Streptococcus spp. assay, the high number of false-positive results in the field samples might be explained by differences in specificity between the reference method and the LAMP assay: the reference method does not report irrelevant growth, such as environmental streptococci that may be present in the milk, but likely are not associated with mastitis. The LAMP assay, however, was developed as a genus assay instead of a species assay and thus detected all Streptococcus spp. This effect is seen more often when culture is compared with DNA techniques such as PCR (Taponen et al., 2009; Koskinen et al., 2010; Oikonomou et al., 2012), and could also have affected the results of $K$. pneumoniae assay as being an abundant pathogen in the environment of the cow (Zadoks et al., 2011). Collecting milk samples is sensitive to contamination, and thus environmental bacteria may be present in the milk, without being associated with mastitis. Thus, proper sample collection is important to obtain reliable test results, but might be even more important when on-site tests will be applied. Nevertheless, this type of misclassification for the Streptococcus spp. LAMP assay would not lead to problems from a treatment perspective in practice, because most samples with false-positive results contained gram-positive bacteria according to the reference method, and thus the treatment applied would probably also affect these gram-positive bacteria. From the perspective of prudent antimicrobial use, however, these misclassifications are unwanted. Thus, to obtain a test that results in relevant outcomes, further research should be performed on more samples collected in the field to determine the criteria necessary to judge samples for the K. pneumoniae and Streptococcus spp. LAMP assay.
We used 3 criteria to judge the results of the LAMP assays: whether a signal emerged within the length of the experiment, the presence of a sigmoidal amplification curve, and the Tm of the amplification product. The Tm of amplicons is determined by length and composition of the amplicon, and in part by the amount of template DNA and the amount of dye in a reaction (Monis et al., 2005; Reed et al., 2007), and differs among different strains of the same species (Guion et al., 2008). Thus, if Tm is used as a judgment criterion, a range rather than a single Tm is demanded. Only 1 positive control strain was used to determine the Tm of the LAMP products. As the variation of the products in field strains is not known, an arbitrary $2^{\circ} \mathrm{C}$ margin around the mean was used. It is conceivable that with a larger set of strains this range can be fine tuned. Thus, further research is necessary to better understand the effects of the different criteria used, specifically to judge the results of the K. pneumoniae and Streptococcus spp. assay on multiple bacterial species.

All LAMP reactions were performed in a real-time PCR machine. However, the ultimate goal is to develop a test that can be used on-site. For that, all steps, from sample preparation to the readout of the result, should be easy to execute with minimal and simple equipment. As a first step toward on-site LAMP, the feasibility of NALFIA as readout was investigated; isolating DNA and performing of the assay was still done with protocols and equipment that require a laboratory. We found that the limit of detection was comparable between the real-time and NALFIA readout. From a practical perspective, we chose to work with a generic runtime of $40 \mathrm{~min}$ for all LAMP reactions as to make the test suitable for parallel on-site testing. When tested with the selected milk samples, the NALFIA readout had an almost perfect agreement with the reference method, except for $K$. pneumoniae with a kappa of 0.40 . Most of the samples with false-positive results in the $K$. pneumoniae assay contained $E$. coli according to the reference method. Thus, if the K. pneumoniae NALFIA assay would be used in the field, E. coli cases would be pointed as $K$. pneumoniae cases, which involves different cure outcomes and thus likely different approaches are to be expected (Fuenzalida and Ruegg, 2019). Nevertheless, if NALFIA would be used as readout, as for every endpoint readout, the reaction should be stopped at the optimal $\mathrm{Tp}$ cut-off to obtain optimal results. Moreover, as ideally the assay should be run in multiplex on the same sample and for practical reasons a generic runtime is preferred, new designs are necessary for the K. pneumoniae and Streptococcus spp. assay.

We aimed to develop fast, on-site tests to detect mastitis pathogens. Whether molecular tests are suitable for on-farm use can be disputed. For example, results of 
molecular tests need to be interpreted differently than culture results, and training might be more demanding regarding the potential higher expertise levels required. Because molecular tests could provide a result within the requested time to result of $8 \mathrm{~h}$, where culture cannot, such tests are of interest to further study their application possibilities. In the current evaluation, 3 technical issues remain that make the current method not yet suitable for on-site use: the DNA extraction, the equipment, and a practical approach to test all LAMP assays in parallel on DNA of the same sample. First, although LAMP in itself is a fast test, the time needed to extract DNA from milk as performed in this study was time consuming and resulted in a total time to result of about 6 to $8 \mathrm{~h}$ for the LAMP assays. Nevertheless, that is faster than culture and lies within the time to result indicated as preferred by farmers (Griffioen et al., 2016). However, LAMP generally is described as a test that is less demanding in terms of DNA purification than real-time PCR. For example, diluted virus stocks were directly added to the reaction mix to perform the LAMP reaction or centrifuged pellet samples were boiled with Triton X-100 after which the lysates were used for the LAMP reactions (Kaneko et al., 2007; Sowmya et al., 2012). Therefore, simple, somewhat crude methods for sample preparation might suffice for LAMP (Cremonesi et al., 2006; Cressier and Bissonnette, 2011; Sowmya et al., 2012; Lee et al., 2019), also for mastitis milk samples. Further research is necessary to find a simplified sample preparation without deteriorating test characteristics. Second, different studies report on the possibility of a less expensive or portable instrument for running the amplifications (Lee et al., 2019; Wilisiani et al., 2019) to be used on-site (Tangkanchanapas et al., 2018). We performed preliminary experiments with a LAMP assay for $S$. uberis (data not shown) using a portable battery-powered instrument designed for performing on-site LAMP (Genie II, Optigene Ltd., Horsham, West Sussex, UK), which resulted in similar results as described (Cornelissen et al., 2016). As LAMP reactions are performed at a constant temperature, even a simple heating block will be sufficient to perform the LAMP reaction (Tangkanchanapas et al., 2018). Furthermore, additional control criteria on Tm or sigmoidal curve to judge the results are not available in an on-site readout like NALFIA. Thus, it is important to thoroughly evaluate the designed assays to know the effects in the field. Generally, a species assay may be preferred over a genus assay to avoid high numbers of false-positive results, as explained for the Streptococcus spp. LAMP assay. Because the results we found were comparable between both readouts, ignoring additional criteria like the Tm and sigmoidal amplification curve could be considered. To assess the performance of these LAMP assays with a simple DNA extraction protocol and a heating block would be the next step to develop on-site tests for mastitis-causing pathogens.

In conclusion, LAMP is a promising method for fast on-site tests for mastitis-causing pathogens. The E. coli and Staph. aureus had a kappa $\geq 0.75$ with the reference method. For the K. pneumoniae and Streptococcus spp., however, the design was suboptimal. As a consequence, K. pneumoniae in the NALFIA readout and the Streptococcus spp. assay on the field samples resulted in a high number of false-positive results. Nevertheless, the NALFIA is an easy and reliable readout for on-site use as the results were similar to the results obtained using the real-time readout, with that remark that a shorter runtime needs to be performed for the $K$. pneumoniae assay. The current LAMP designs demonstrate the capability of LAMP for detecting mastitis pathogens. Optimized designs and reaction conditions may improve the performance of this type of LAMP assay, and when a simplified sample preparation protocol becomes available, the combination of LAMP and NALFIA has the potential to enable fast and reliable on-site testing on milk from cows with clinical mastitis.

\section{ACKNOWLEDGMENTS}

This study was financed by ZuivelNL (DairyNL, The Hague, the Netherlands) and the Ministry of Agriculture, Nature and Food Quality in the 1Health4Food public-private partnership (TKI-AF 12067) in the project "Diagnostiekontwikkeling en -toepassing voor het optimaliseren van uiergezondheid" executed by the Dutch Mastitis Diagnostics Consortium. We thank all farmers who participated in the field study for sample collection. Additionally, we thank the bacteriology staff of Royal GD. The authors have not stated any conflicts of interest.

\section{REFERENCES}

Appelt, S., S. S. Aly, K. Tonooka, K. Glenn, Z. Xue, T. W. Lehenbauer, and M. L. Marco. 2019. Development and comparison of loop-mediated isothermal amplification and quantitative polymerase chain reaction assays for the detection of Mycoplasma bovis in milk. J. Dairy Sci. 102:1985-1996. https://doi.org/10.3168/jds .2018-15306.

Ashraf, A., and M. Imran. 2018. Diagnosis of bovine mastitis: From laboratory to farm. Trop. Anim. Health Prod. 50:1193-1202. https://doi.org/10.1007/s11250-018-1629-0.

Barreiro, J. R., C. R. Ferreira, G. B. Sanvido, M. Kostrzewa, T. Maier, B. Wegemann, V. Böttcher, M. N. Eberlin, and M. V. dos Santos. 2010. Short communication: Identification of subclinical cow mastitis pathogens in milk by matrix-assisted laser desorption/ioniza- 
tion time-of-flight mass spectrometry. J. Dairy Sci. 93:5661-5667. https://doi.org/10.3168/jds.2010-3614.

Byrt, T., J. Bishop, and J. B. Carlin. 1993. Bias, prevalence and kappa. J. Clin. Epidemiol. 46:423-429. https://doi.org/10.1016/0895 $-4356(93) 90018-\mathrm{V}$.

Caraguel, C. G. B., H. Stryhn, N. Gagné, I. R. Dohoo, and K. L. Hammell. 2011. Selection of a cutoff value for real-time polymerase chain reaction results to fit a diagnostic purpose: Analytical and epidemiologic approaches. J. Vet. Diagn. Invest. 23:2-15. https:// doi.org/10.1177/104063871102300102.

Cornelissen, J. B. W. J., A. De Greeff, A. E. Heuvelink, M. Swarts, H. E. Smith, and F. J. Van der Wal. 2016. Rapid detection of Streptococcus uberis in raw milk by loop-mediated isothermal amplification. J. Dairy Sci. 99:4270-4281. https://doi.org/10.3168/ jds.2015-10683.

Cremonesi, P., B. Castiglioni, G. Malferrari, I. Biunno, C. Vimercati, P. Moroni, S. Morandi, and M. Luzzana. 2006. Technical Note: Improved method for rapid DNA extraction of mastitis pathogens directly from milk. J. Dairy Sci. 89:163-169. https://doi.org/10 .3168/jds.S0022-0302(06)72080-X.

Cressier, B., and N. Bissonnette. 2011. Assessment of an extraction protocol to detect the major mastitis-causing pathogens in bovine milk. J. Dairy Sci. 94:2171-2184. https://doi.org/10.3168/jds.2010 -3669 .

Dohoo, I. R., W. Martin, and H. Stryhn. 2009. Screening and diagnostic tests. Pages 91-134 in Veterinary Epidemiologic Research. 2nd ed. VER Inc., Charlottetown, Prince Edward Island, Canada.

Duarte, C. M., P. P. Freitas, and R. Bexiga. 2015. Technological advances in bovine mastitis diagnosis: An overview. J. Vet. Diagn. Invest. 27:665-672. https://doi.org/10.1177/1040638715603087.

Fuenzalida, M. J., and P. L. Ruegg. 2019. Negatively controlled, randomized clinical trial to evaluate intramammary treatment of nonsevere, gram-negative clinical mastitis. J. Dairy Sci. 102:54385457. https://doi.org/10.3168/jds.2018-16156.

Griffioen, K., G. E. Hop, M. M. C. Holstege, A. G. J. Velthuis, T. J. G. M. Lam, and 1Health4Food-Dutch Mastitis Diagnostics Consortium. 2016. Dutch dairy farmers' need for microbiological mastitis diagnostics. J. Dairy Sci. 99:5551-5561. https://doi.org/10.3168/ jds.2015-10816.

Griffioen, K., A. G. J. Velthuis, L. A. Lagerwerf, A. E. Heuvelink, and T. J. G. M. Lam. 2018. Agreement between four commercial diagnostic tests and routine bacteriological culture of milk to determine the udder infection status of dairy cows. Prev. Vet. Med. 157:162-173. https://doi.org/10.1016/j.prevetmed.2018.07.003.

Guion, C. E., T. J. Ochoa, C. M. Walker, F. Barletta, and T. G. Cleary. 2008. Detection of diarrheagenic Escherichia coli by use of melting-curve analysis and real-time multiplex PCR. J. Clin. Microbiol. 46:1752-1757. https://doi.org/10.1128/JCM.02341-07.

Kaneko, H., T. Kawana, E. Fukushima, and T. Suzutani. 2007. Tolerance of loop-mediated isothermal amplification to a culture medium and biological substances. J. Biochem. Biophys. Methods 70:499-501. https://doi.org/10.1016/j.jbbm.2006.08.008.

Koskinen, M. T., J. Holopainen, S. Pyorala, P. Bredbacka, A. Pitkala, H. W. Barkema, R. Bexiga, J. Roberson, L. Solverod, R. Piccinini, D. Kelton, H. Lehmusto, S. Niskala, and L. Salmikivi. 2009. Analytical specificity and sensitivity of a real-time polymerase chain reaction assay for identification of bovine mastitis pathogens. J. Dairy Sci. 92:952-959. https://doi.org/10.3168/jds.2008-1549.

Koskinen, M. T., G. J. Wellenberg, O. C. Sampimon, J. Holopainen, A. Rothkamp, L. Salmikivi, W. A. van Haeringen, T. J. G. M. Lam, and S. Pyörälä. 2010. Field comparison of real-time polymerase chain reaction and bacterial culture for identification of bovine mastitis bacteria. J. Dairy Sci. 93:5707-5715. https://doi .org/10.3168/jds.2010-3167.

Kuipers, A., W. J. Koops, and H. Wemmenhove. 2016. Antibiotic use in dairy herds in the Netherlands from 2005 to 2012. J. Dairy Sci 99:1632-1648. https://doi.org/10.3168/jds.2014-8428.

Lam, T., R. Olde Riekerink, O. C. Sampimon, and H. Smith. 2009. Mastitis diagnostics and performance monitoring: A practical approach. Ir. Vet. J. 62(S4):34-39. https://doi.org/10.1186/2046 -0481-62-S4-S34.
Landis, J. R., and G. G. Koch. 1977. The measurement of observer agreement for categorical data. Biometrics 33:159-174. https://doi .org/10.2307/2529310.

Lee, S., V. S. L. Khoo, C. A. D. Medriano, T. Lee, S.-Y. Park, and S. Bae. 2019. Rapid and in situ detection of fecal indicator bacteria in water using simple DNA extraction and portable loop-mediated isothermal amplification (LAMP) PCR methods. Water Res. 160:371-379. https://doi.org/10.1016/j.watres.2019.05.049.

Mahmmod, Y. S., N. Toft, J. Katholm, C. Gronbaek, and I. C. Klaas. 2013. Estimation of test characteristics of real-time PCR and bacterial culture for diagnosis of subclinical intramammary infections with Streptococcus agalactiae in Danish dairy cattle in 2012 using latent class analysis. Prev. Vet. Med. 109:264-270. https://doi .org/10.1016/j.prevetmed.2012.10.018.

Monis, P. T., S. Giglio, and C. P. Saint. 2005. Comparison of SYT09 and SYBR Green I for real-time polymerase chain reaction and investigation of the effect of dye concentration on amplification and DNA melting curve analysis. Anal. Biochem. 340:24-34. https:// doi.org/10.1016/j.ab.2005.01.046.

Mori, Y., and T. Notomi. 2009. Loop-mediated isothermal amplification (LAMP): a rapid, accurate, and cost-effective diagnostic method for infectious diseases. J. Infect. Chemother. 15:62-69. https://doi.org/10.1007/s10156-009-0669-9.

NMC. 1999. Laboratory Handbook on Bovine Mastitis. National Mastitis Council, Madison, WI.

Oikonomou, G., V. S. Machado, C. Santisteban, Y. H. Schukken, and R. C. Bicalho. 2012. Microbial diversity of bovine mastitic milk as described by pyrosequencing of metagenomic $16 \mathrm{~S}$ rDNA. PLoS One 7:e47671. https://doi.org/10.1371/journal.pone.0047671.

Pol, M., and P. L. Ruegg. 2007. Treatment practices and quantification of antimicrobial drug usage in conventional and organic dairy farms in Wisconsin. J. Dairy Sci. 90:249-261. https://doi.org/10 $.3168 /$ jds.S0022-0302(07)72626-7.

Reed, G. H., J. O. Kent, and C. T. Wittwer. 2007. High-resolution DNA melting analysis for simple and efficient molecular diagnostics. Pharmacogenomics 8:597-608. https://doi.org/10.2217/ 14622416.8.6.597.

Ruegg, P. L. 2018. Making antibiotic treatment decisions for clinical mastitis. Vet. Clin. North Am. Food Anim. Pract. 34:413-425. https://doi.org/10.1016/j.cvfa.2018.06.002.

Sange, M. D., A. Becker, A. A. Hassan, M. Bülte, M. Ganter, U. Siebert, and A. Abdulmawjood. 2019. Development and validation of a loop-mediated isothermal amplification assay-A rapid and sensitive detection tool for Mycobacterium avium ssp. paratuberculosis in small ruminants. J. Appl. Microbiol. 127:47-58. https:// doi.org/10.1111/jam.14284.

Sathish, G., E. Hemakumar, and K. C. Divya. 2016. Rapid detection of MRSA by loop mediated isothermal amplification in bovine milk samples. Br. Microbiol. Res. J. 17:1-5. https://doi.org/10 .9734/BMRJ/2016/28037.

Sears, P. M., and K. K. McCarthy. 2003. Diagnosis of mastitis for therapy decisions. Vet. Clin. North Am. Food Anim. Pract. 19:93-108. https://doi.org/10.1016/S0749-0720(02)00074-9.

Sheet, O. H., N. T. Grabowski, G. Klein, and A. Abdulmawjood. 2016. Development and validation of a loop mediated isothermal amplification (LAMP) assay for the detection of Staphylococcus aureus in bovine mastitis milk samples. Mol. Cell. Probes 30:320-325. https: //doi.org/10.1016/j.mcp.2016.08.001.

Shome, B. R., S. D. Mitra, M. Bhuvana, N. Krithiga, D. Velu, R. Shome, S. Isloor, S. B. Barbuddhe, and H. Rahman. 2011. Multiplex PCR assay for species identification of bovine mastitis pathogens. J. Appl. Microbiol. 111:1349-1356. https://doi.org/10.1111/ j.1365-2672.2011.05169.x.

Song, L., J. Li, S. Hou, X. Li, and S. Chen. 2012. Establishment of loop-mediated isothermal amplification (LAMP) for rapid detection of Brucella spp. and application to milk an blood samples. J. Microbiol. Methods 90:292-297. https://doi.org/10.1016/j.mimet .2012.05.024.

Sowmya, N., M. S. Thakur, and H. K. Manonmani. 2012. Rapid and simple DNA extraction method for the detection of enterotoxigenic Staphylococcus aureus directly from food samples: Comparison 
of PCR and LAMP methods. J. Appl. Microbiol. 113:106-113. https://doi.org/10.1111/j.1365-2672.2012.05315.x.

Tangkanchanapas, P., M. Höfte, and K. D. Jonghe. 2018. Reverse transcription loop-mediated isothermal amplification (RT-LAMP) designed for fast and sensitive on-site detection of Pepper chat fruit viroid (PCFVd). J. Virol. Methods 259:81-91. https://doi.org/10 .1016/j.jviromet.2018.06.003.

Taponen, S., L. Salmikivi, H. Simojoki, M. T. Koskinen, and S. Pyörälä. 2009. Real-time polymerase chain reaction-based identification of bacteria in milk samples from bovine clinical mastitis with no growth in conventional culturing. J. Dairy Sci. 92:26102617. https://doi.org/10.3168/jds.2008-1729.

Tian, X., J. Feng, and Y. Wang. 2018. Direct loop-mediated isothermal amplification assay for on-site detection of Staphylococcus aureus. FEMS Microbiol. Lett. 365:1-6. https://doi.org/10.1093/ femsle/fny092.

Tie, Z., W. Chunguang, W. Xiaoyuan, Z. Xingua, and Z. Xiuhui. 2012. Loop-mediated isothermal amplification for detection of Staphylococcus aureus in dairy cow suffering from mastitis. J. Biomed. Biotechnol. 2012:435982.
Wang, D., and Y. Liu. 2015. Development of primer sets for loopmediated isothermal amplification that enables rapid and specific detection of Streptococcus dysgalactiae, Streptococcus uberis and Streptococcus agalactiae. Int. J. Environ. Res. Public Health 12:5735-5742. https://doi.org/10.3390/ijerph120605735.

Wilisiani, F., A. Tomiyama, H. Katoh, S. Hartono, Y. Neriya, H. Nishigawa, and T. Natsuaki. 2019. Development of a LAMP assay with a portable device for real-time detection of begomoviruses under field conditions. J. Virol. Methods 265:71-76. https://doi.org/10 $.1016 /$ j.jviromet.2018.10.005.

Yang, W., X. Song, J. Wang, Z. Li, M. Ji, and Y. Li. 2014. Detection methods for milk pathogenic bacteria by loop-mediated isothermal amplification. Biosci. Trends 8:316-321. https://doi.org/10.5582/ bst.2014.01118.

Zadoks, R. N., H. M. Griffiths, M. A. Munoz, C. Ahlstrom, G. J. Bennett, E. Thomas, and Y. H. Schukken. 2011. Sources of Klebsielle and Raoultella species on dairy farms: Be careful where you walk. J. Dairy Sci. 94:1045-1051. https://doi.org/10.3168/jds.2010-3603.

Zamani, A., R. Y. Mashouf, A. E. Namvar, and M. Y. Alikhani. 2013. Detection of magA gene in Klebsiella spp. isolated from clinical samples. Iran. J. Basic Med. Sci. 16:173-176. 